\title{
Ultrasound measurement of pediatric visceral fat thickness: correlations with metabolic and liver profiles
}

\author{
Jae Hwa Jung, MD', \\ Mo Kyung Jung, MD', \\ Ki Eun Kim, MD', \\ Ah Reum Kwon, MD', \\ Hyun Wook Chae, MD', \\ Choon Sik Yoon, MD, PhD', \\ Ho Seong Kim, MD, PhD', \\ Duk-Hee Kim, MD, PhD ${ }^{3}$
}

Departments of ${ }^{1}$ Pediatrics and ${ }^{2}$ Radiology, Yonsei University College of Medicine, Seoul, ${ }^{3}$ Sowha Children's Hospital, Seoul, Korea

Received: 24 October, 2015

Revised: 28 October, 2015

Accepted: 30 November, 2015

Address for correspondence:

Hyun-Wook Chae, MD

Department of Pediatrics, Gangnam Severance Hospital, Yonsei University College of Medicine, 50-1 Yonsei-ro, Seodaemun-gu, Seoul 03722, Korea Tel: +82-2-2019-3355,

Fax: +82-2-3461-9473,

E-mail: hopechae@yuhs.ac
Purpose: Abdominal obesity is a fundamental factor underlying the development of metabolic syndrome. Because of radiation exposure and cost, computed tomography or dual-energy X-ray absorptiometry to evaluate abdominal adiposity are not appropriate in children. Authors evaluated whether ultrasound results could be an indicator of insulin resistance and nonalcoholic fatty liver disease (NAFLD).

Methods: We enrolled 73 subjects (aged 6-16 years) who were evaluated abdominal adiposity by ultrasound. Subcutaneous fat thickness was defined as the measurement from the skin-fat interface to the linea alba, and visceral fat thickness (VFT) was defined as the thickness from the linea alba to the aorta. Anthropometric and biochemical metabolic parameters were also collected and compared. The subjects who met 2 criteria, radiologic confirmed fatty liver and alanine aminotransferase $>40$, were diagnosed with NAFLD.

Results: There was a strong positive correlation between VFT and obesity. VFT was highly correlated with the homeostasis model assessment for insulin resistance score $(r=0.403, P<0.001)$. The area under the curve for VFT as a predictor of NAFLD was 0.875 (95\% confidence interval [Cl], 0.787-0.964). VFT of $34.3 \mathrm{~mm}$ was found to be the discriminating cutoff for NAFLD (sensitivity, $84.6 \%$; specificity, $71.2 \%$, respectively).

Conclusion: Ultrasound could be useful in measuring VFT and assessing abdominal adiposity in children. Moreover, increased VFT might be an appropriate prognostic factor for insulin resistance and NAFLD.

Keywords: Intra-abdominal fat, Ultrasonography, Child, Abdominal obesity metabolic syndrome, Fatty liver

\section{Introduction}

In recent years, obesity has become a major public health and medical care problem. The rates of overweight and obese children and adolescents continue to increase rapidly, and the World Health Organization classifies obesity as a disease ${ }^{1)}$. The prevalence of childhood obesity has also increased in Korea ${ }^{2}$. The prevalence of obesity in 10- to 14-year-old males in Seoul has increased 10 fold from 1.7\% to $17.9 \%$ between 1999 and 2002. This is as high as the $14 \%-17 \%$ rate in the United States ${ }^{3,4)}$.

Obesity is closely associated with insulin resistance, dyslipidemia, hypertension, cardiovascular disease, and fatty liver. Hemodynamic and metabolic changes are related and play important roles in the development of metabolic syndrome ${ }^{5}$. The amount of body fat itself is a risk factor; however, body fat distribution, particularly abdominal obesity, is a crucial risk factor for metabolic syndrome and cardiovascular disease ${ }^{6,7}$. Furthermore, the prevalence of nonalcoholic fatty liver disease (NAFLD), which is linked with high abdominal 
fat, is increasing in children. Thus, identifying and measuring abdominal fat content are important for evaluating obesity in this vulnerable population.

Computed tomography (CT) and dual-energy X-ray absorptiometry (DEXA) are commonly used methods to measure abdominal obesity in adults. However, the high cost and radiation exposure make them challenging to use in children and adolescents in clinical settings ${ }^{8,9)}$.

Since Armellini et al. ${ }^{10)}$ first suggested the utility of ultrasound to measure abdominal fat in 1990, many studies have employed this method ${ }^{11)}$. One study reported a high correlation for abdominal fat measurement between ultrasound and $\mathrm{CT}^{12)}$. Although ultrasound might be a good method to replace CT in adults, there are few studies in pediatric populations.

In this study, we investigated the utility of ultrasound in measuring abdominal fat and the relationship of this value with obesity indicators. We also evaluated whether the results of ultrasound could be an indicator of insulin resistance and NAFLD.

\section{Materials and methods}

We retrospectively analyzed data from 73 subjects (aged 6-16 years) who visited the Gangnam Severance Hospital, Yonsei University between January 2013 and December 2014, for obesity and growth assessment that included ultrasound and blood testing. We excluded patients who might have diseases that can interrupt normal growth. This study was approved by the Institutional Review Board of Yonsei University Gangnam Severance Hospital (approval number: 3-2015-0099).

We performed ultrasound evaluation to measure abdominal obesity, including visceral fat thickness (VFT) and subcutaneous fat thickness (SFT). Ultrasonography was performed with a high-resolution ultrasonographic system (SA 9900; Medison, Seoul, Korea). VFT was defined as the thickness of fat tissue between the linea alba and aorta, and SFT was defined as fat tissue thickness from the skin-fat interface to the linea alba. Transverse scanning was performed to measure the maximum SFT and VFT using 7.5- and 3.5-MHz probes, respectively. Both measures were obtained $1 \mathrm{~cm}$ above the umbilicus by the same pediatric radiologist.

Anthropometric data, and biochemical measurements were collected by nurse. Participants underwent a standard examination and testing including measurement of height, weight, body mass index (BMI), lipid profiles, aspartate aminotransferase (AST), alanine aminotransferase (ALT), protein, and albumin. The height and weight were measured to the nearest $0.1 \mathrm{~cm}$ and $0.1 \mathrm{~kg}$, respectively, while the subjects were wearing light clothing and not wearing shoes. The waist circumference was measured at the midpoint between the lateral iliac crest and the lowest rib. The homeostasis model assessment for insulin resistance (HOMA-IR) score was used as an indicator of insulin resistance ${ }^{13)}$. We defined standard deviation score for height, weight, and BMI based on the 2007 Korean National Growth Charts ${ }^{14)}$. The subjects who met 2 criteria, radiologic confirmed fatty liver and ALT $>40$, were diagnosed with NAFLD. We divided the participants into 3 groups. BMI percentiles above the 95th percentile are labeled 'overweight,' BMI percentiles between the 85 th and 95 th percentiles are labeled 'at risk for overweight', and BMI percentiles below the 85 th $^{\text {are }}$ 'normal' ${ }^{15)}$.

Statistical analyses were performed with SAS ver. 9.2 (SAS Institute, Cary, NC, USA). Associations between continuous variables were determined using Pearson correlation coefficients. Intergroup comparisons were performed with independentsample $t$-tests. Multiple regression tests were used to verify the association of VFT and insulin resistance. Analyses of variance and Fisher exact tests were used to compare the 3 groups. A receiver operating characteristic (ROC) curve was used to determine the appropriate VFT cutoff value. Differences were considered significant at $P<0.05$.

\section{Results}

We analyzed the baseline clinical and biochemical parameters, and Table 1 shows the mean and standard deviation values for the 47 males and 26 females enrolled in this study. The mean BMI $z$-score of the participants was 1.7 , indicating that most

Table 1. Anthropometric data $(\mathbf{n}=73)$

\begin{tabular}{|c|c|}
\hline Parameter & Value \\
\hline Age (yr) & $10.3 \pm 2.3$ \\
\hline \multicolumn{2}{|l|}{ Sex } \\
\hline Male:female & $47: 26$ \\
\hline Height z-score & $1.1 \pm 1.1$ \\
\hline Weight z-score & $1.8 \pm 0.7$ \\
\hline BMI z-score & $1.7 \pm 0.6$ \\
\hline Glucose (mg/dL) & $98.1 \pm 13.5$ \\
\hline AST (IU/L) & $28.6 \pm 11.2$ \\
\hline ALT (IU/L) & $31.5 \pm 25.4$ \\
\hline Cholesterol (mg/dL) & $182.7 \pm 31.6$ \\
\hline $\mathrm{TG}(\mathrm{mg} / \mathrm{dL})$ & $138.7 \pm 90.0$ \\
\hline LDL (mg/dL) & $102.6 \pm 20.3$ \\
\hline $\mathrm{HDL}(\mathrm{mg} / \mathrm{dL})$ & $49.3 \pm 9.1$ \\
\hline Insulin ( $\mu \mathrm{U} / \mathrm{mL})$ & $17.0 \pm 7.6$ \\
\hline CRP (mg/L) & $1.9 \pm 1.5$ \\
\hline Abdomen circumference $(\mathrm{cm})$ & $88.2 \pm 9.4$ \\
\hline VFT (mm) & $32.6 \pm 10.6$ \\
\hline $\mathrm{SFT}(\mathrm{mm})$ & $20.1 \pm 7.6$ \\
\hline VFT:SFT ratio & $1.8 \pm 0.7$ \\
\hline Fatty liver (n) & 30 \\
\hline HOMA-IR & $4.2 \pm 2.1$ \\
\hline
\end{tabular}

Values are presented as mean \pm standard deviation unless otherwise indicated.

BMI, body mass index; AST, aspartate aminotransferase; ALT, alanine aminotransferase; TG, triglycerides; LDL, low-density lipoprotein; HDL, high-density lipoprotein; CRP, C-reactive protein; VFT, visceral fat thickness; SFT, subcutaneous fat thickness; HOMA-IR, homeostasis model assessment for insulin resistance. 
Table 2. Comparison of clinical characteristics among the three BMI percentile groups

\begin{tabular}{|c|c|c|c|c|c|}
\hline Variable & $\begin{array}{l}\text { BMI <85th percentile } \\
(n=9)\end{array}$ & $\begin{array}{l}\text { BMI, 85th-95th percentile } \\
\qquad(n=27)\end{array}$ & $\begin{array}{l}\text { BMI >95th percentile } \\
\quad(n=37)\end{array}$ & $P$-value & $P$ for trend \\
\hline Glucose (mg/dL) & $101.1 \pm 13.1$ & $95.8 \pm 5.9$ & $99.1 \pm 17.2$ & 0.495 & 0.684 \\
\hline AST (IU/L) & $27.1 \pm 5.0$ & $26.0 \pm 9.3$ & $30.9 \pm 13.1$ & 0.197 & 0.358 \\
\hline ALT (IU/L) & $23.0 \pm 18.2$ & $24.8 \pm 16.9$ & $39.4 \pm 29.2$ & $0.024^{*}$ & 0.011 \\
\hline Cholesterol (mg/dL) & $184.9 \pm 16.0$ & $184.7 \pm 31.7$ & $180.7 \pm 34.8$ & 0.861 & 0.725 \\
\hline $\mathrm{TG}(\mathrm{mg} / \mathrm{dL})$ & $106.0 \pm 44.0$ & $130.7 \pm 73.7$ & $152.5 \pm 106.4$ & 0.326 & 0.168 \\
\hline $\mathrm{HDL}(\mathrm{mg} / \mathrm{dL})$ & $51.6 \pm 10.0$ & $51.2 \pm 9.5$ & $47.5 \pm 8.6$ & 0.233 & 0.238 \\
\hline LDL (mg/dL) & $100.3 \pm 7.8$ & $103.4 \pm 23.0$ & $103.0 \pm 24.7$ & 0.842 & 0.971 \\
\hline Insulin ( $\mu \mathrm{U} / \mathrm{mL})$ & $13.0 \pm 5.4$ & $14.5 \pm 6.2$ & $19.9 \pm 8.0$ & $0.004^{*}$ & 0.011 \\
\hline CRP (mg/L) & $0.5 \pm 0.4$ & $1.5 \pm 1.4$ & $2.3 \pm 1.5$ & 0.120 & 0.093 \\
\hline Abdomen circumference (cm) & $78.5 \pm 4.8$ & $86.7 \pm 7.7$ & $95.4 \pm 9.1$ & $0.003^{*}$ & 0.001 \\
\hline $\mathrm{VFT}(\mathrm{mm})$ & $27.3 \pm 10.5$ & $28.5 \pm 7.3$ & $37.0 \pm 11.0$ & $0.001^{*}$ & 0.009 \\
\hline $\mathrm{SFT}(\mathrm{mm})$ & $14.4 \pm 5.5$ & $17.8 \pm 5.9$ & $23.0 \pm 7.9$ & $0.009^{*}$ & 0.009 \\
\hline No. of fatty liver (\%) & $2(22.2)$ & $7(25.9)$ & $21(56.8)$ & $0.024^{\dagger}$ & - \\
\hline HOMA-IR & $3.4 \pm 1.9$ & $3.5 \pm 1.5$ & $4.9 \pm 2.3$ & $0.008^{*}$ & 0.037 \\
\hline
\end{tabular}

Values are presented as mean \pm standard deviation unless otherwise indicated.

BMI, body mass index; AST, aspartate aminotransferase; ALT, alanine aminotransferase; TG, triglycerides; LDL, low-density lipoprotein; HDL, high-density lipoprotein; CRP, C-reactive protein; VFT, visceral fat thickness; SFT, subcutaneous fat thickness; HOMA-IR, homeostasis model assessment for insulin resistance.

*Significantly different from analysis of variance test: $P<0.05$. 'Significantly different from Fisher exact tests: $P<0.05$

Table 3. Pearson correlation coefficients of biochemical factors to VFT

\begin{tabular}{lrr}
\hline \multirow{2}{*}{ Parameter } & \multicolumn{2}{c}{ VFT } \\
\cline { 2 - 3 } & \multicolumn{1}{c}{$r$} & P-value \\
\hline Glucose $(\mathrm{mg} / \mathrm{dL})$ & 0.055 & 0.644 \\
BMl z-score & 0.505 & $<0.001$ \\
AST (IU/L) & 0.247 & 0.037 \\
ALT (IU/L) & 0.411 & $<0.001$ \\
Cholesterol (mg/dL) & 0.098 & 0.415 \\
TG (mg/dL) & 0.272 & 0.021 \\
HDL (mg/dL) & -0.247 & 0.037 \\
Insulin $(\mu \mathrm{U} / \mathrm{mL})$ & 0.436 & $<0.001$ \\
CRP $(\mathrm{mg} / \mathrm{L})$ & 0.212 & 0.236 \\
Abdomen circumference (cm) & 0.635 & $<0.001$ \\
SFT (mm) & 0.325 & 0.021 \\
HOMA-IR & 0.403 & $<0.001$ \\
\hline VFT, VIscerl fat thickness; BMI, body
\end{tabular}

VFT, visceral fat thickness; BMI, body mass index; AST, aspartate aminotransferase; ALT, alanine aminotransferase; TG, triglycerides; LDL, low-density lipoprotein; HDL, high-density lipoprotein; CRP, C-reactive protein; SFT, subcutaneous fat thickness; HOMA-IR, homeostasis model assessment for insulin resistance.

subjects were overweight or obese.

The subjects were classified into 3 groups based on BMI percentile groups (Table 2). We identified the increasing trends of ALT, insulin, abdomen circumference, VFT, SFT, and HOMAIR in 3 groups. There were no significant differences in glucose and high-density lipoprotein (HDL) among the 3 groups. The number of fatty liver was 2 of $9(22.2 \%)$ in BMI $<85$ th percentile group, 7 of 27 (25.9\%) in BMI 85th-95th percentile group, and 21 of $37(56.8 \%)$ in BMI $>95$ th percentile group $(P=0.024)$. We also observed a significant difference of HOMA-IR score, which represented insulin resistance $(P=0.008)$.
Table 4. Multiple regression analysis variances of biochemical factors explaining HOMA-IR

\begin{tabular}{lccc}
\hline \multirow{2}{*}{ Variable } & \multicolumn{3}{c}{ HOMA-IR } \\
\cline { 2 - 4 } & Beta $(\beta)$ & SE $(\beta)$ & P-value \\
\hline Age & 0.896 & 0.855 & 0.022 \\
ALT & -0.002 & -0.031 & 0.897 \\
BMl z-score & 3.549 & 0.889 & 0.024 \\
Abdomen circumference & -0.249 & -1.123 & 0.016 \\
VFT & 0.054 & 0.271 & 0.048 \\
\hline
\end{tabular}

HOMA-IR, homeostasis model assessment for insulin resistance; $\mathrm{SE}$, standard error; ALT, alanine aminotransferase; BMI, body mass index; VFT, visceral fat thickness.

Table 3 shows the Pearson correlation coefficients between VFT and biochemical factors. As well as BMI $z$-score, most biochemical data were highly correlated with VFT. The measures most highly correlated with VFT were ALT, insulin, and HOMA-IR.

The variances of biochemical factors explaining HOMA-IR obtained with multiple regression analysis are shown in Table 4 . Age $(P=0.022)$, BMI $z$-score $(P=0.024)$, abdomen circumference $(P=0.016)$, VFT $(P=0.048)$ were independent determinants of HOMA-IR $\left(R^{2}\right.$ of the model $\left.=0.278\right)$.

The area under the ROC curve for VFT as a predictor of elevated ALT was 0.849 (95\% confidence interval [CI], 0.7350.963). Relative to VFT $32.0 \mathrm{~mm}$, the sensitivity and specificity to predict ALT $>40 \mathrm{IU} / \mathrm{L}$ were $88.2 \%$ and $74.1 \%$, respectively. ROC analysis of VFT to predict the presence of fatty liver yielded an area under the curve of 0.751 (95\% CI, 0.643-0.859). Relative to VFT $31.8 \mathrm{~mm}$, the sensitivity and specificity to diagnose radiologic fatty liver were $71.0 \%$ and $77.5 \%$, respectively. The ROC for VFT as a predictor of NAFLD was 0.875 (95\% CI, $0.787-0.964)$. VFT $34.3 \mathrm{~mm}$, the sensitivity and specificity to 
diagnose NAFLD were $84.6 \%$ and $71.2 \%$ (Fig. 1).

\section{Discussion}

Obesity is increasing at a rapid pace, and related complications are growing problems in both adults and children. BMI is widely used as a standard for determining whether subjects are overweight or obese ${ }^{16)}$. However, BMI does not reflect body fat distribution. Especially in children, BMI does not adequately assess abdominal obesity because the amount of change in body fat varies depending on growth and development according to $\operatorname{age}^{17,18)}$.

Fat distribution is a more important independent risk factor for cardiovascular disease than percent body fat in children and adolescents. A higher fat distribution in the abdomen is associated with increases in insulin resistance, hyperinsulinemia, and triglycerides (TG), decreased HDL cholesterol, high blood pressure, and the risk of cardiovascular disease $^{19-21)}$. Greater central fat deposition is also associated with less favorable plasma lipid and lipoprotein concentrations, high blood pressure, and greater left ventricular mass ${ }^{22,23)}$. Therefore, we evaluated central obesity by measuring VFT with ultrasound and found strong positive correlations with liver enzyme and TG levels, and a negative correlation with HDL levels. These findings are consistent with previous studies ${ }^{24,25)}$. As well as BMI, VFT had high correlations with metabolic biomarkers such as AST, TG, HDL, and serum insulin levels.

A high correlation between central obesity and insulin resistance has been reported ${ }^{26)}$. Adipose tissue modulates

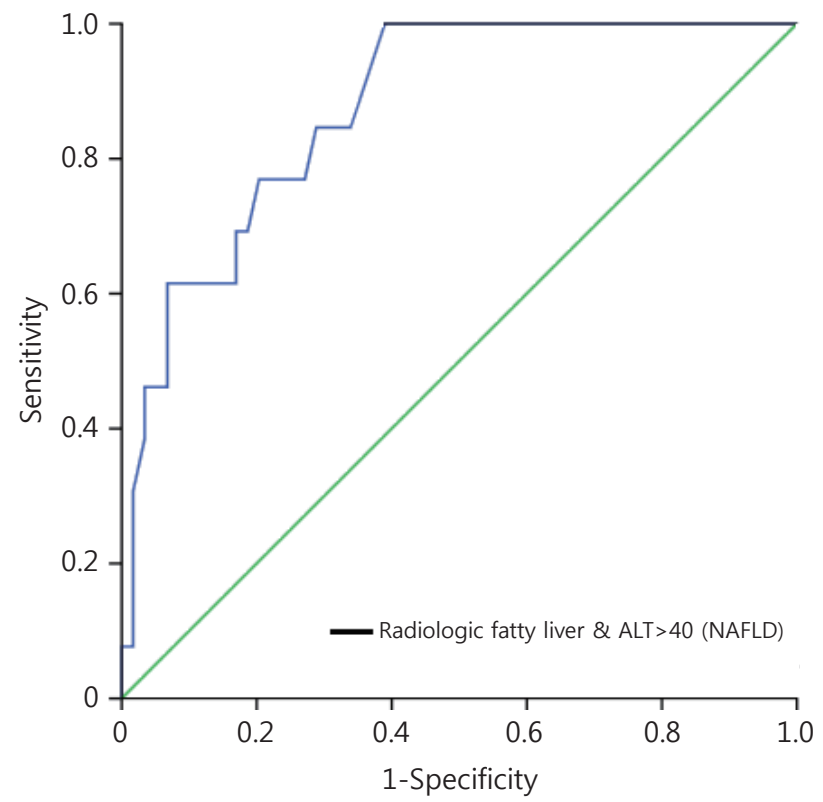

Fig. 1. Receiver operating characteristic (ROC) analysis of visceral fat thickness (VFT) as a predictor of nonalcoholic fatty liver disease (NAFLD). The ROC for VFT as a predictor of NAFLD was 0.875 (95\% confidence interval, $0.787-0.964)$. VFT of $34.3 \mathrm{~mm}$ was found to be the discriminating cutoff for NAFLD (sensitivity, 84.6\%; specificity, $71.2 \%$, respectively). ALT, alanine aminotransferase. metabolism by releasing nonesterified fatty acids, glycerol, hormones including leptin and adiponectin, and proinflammatory cytokines ${ }^{27)}$. The production of these products is increased in the setting of central obesity ${ }^{28)}$. However, insulin resistance also frequently occurs in apparently lean individuals. In a previous study, visceral fat was more strongly correlated with insulin resistance than subcutaneous fat ${ }^{29)}$. In this study, there was also a high correlation between VFT and HOMA-IR, which is an indicator of insulin resistance. Thus, among central obesity measurements, visceral fat accumulation is more useful than other markers.

The rise in the incidence of obesity in children is paralleled by higher rates of fatty liver. NAFLD has become one of the most common chronic hepatopathies in children. Its histologically proven prevalence in children in the United States ranges from $9.6 \%$ in normal-weight individuals up to $38 \%$ in obese subjects ${ }^{30)}$. Nonalcoholic steatohepatitis (NASH) is a subtype of NAFLD accompanied by hepatocyte ballooning and necrosis with or without Mallory's hyaline and fibrosis, and it is potentially progressive in pediatric subjects. Children with NASH may progress to liver cirrhosis and end-stage liver disease with a consequent need for liver transplantation ${ }^{31)}$. Due to its tendency to progress during childhood or after transition into adulthood, early diagnosis and treatment are important. However, NASH is still underdiagnosed in children because the standard confirmatory test is liver biopsy, which has limitations in this population. Ultrasound is the most common imaging technique used for NAFLD screening, but it alone is not sufficient for diagnosis because radiological findings cannot distinguish between NASH and other forms of NAFLD ${ }^{32)}$. We identified a significant relationship between VFT and altered lipid profile, elevated ALT, and insulin resistance. A VFT of 34.3 $\mathrm{mm}$ was a cutoff value to support the diagnosis of NAFLD (sensitivity, 84.6\%; specificity, 71.2\%). Therefore, measuring VFT can be a useful technique to increase NAFLD diagnostic accuracy.

There are several limitations to our analysis. First, because it was a cross-sectional study, the results do not shed light on agerelated changes. Longitudinal analyses are needed to clarify this important issue. Second, our analysis was performed in Korean population, and the VFT cutoff values may differ among ethnic groups. Third, there is a lack of data showing a correlation between VFT and pathologically proven NASH, and comparisons with more established methods such as CT and DXEA. Fourth, by targeting a wide age group, it did not reflect the normal skin and fat thickness variation. Fifth, there was no comparison data about SFT. These issues should be considered when designing future clinical studies.

In conclusion, ultrasound could be useful for measuring abdominal fat thickness and assessing central obesity in children and adolescents. Moreover, ultrasound-measured VFT is an appropriate prognostic factor for ALT elevation, insulin resistance, and NAFLD. Although measuring VFT by ultrasound is not sufficient to determine if a subject has metabolic syndrome or NASH, our results demonstrate that the addition 
of ultrasound-measured VFT significantly improved the prediction of metabolic changes or NAFLD. Ultrasonography is an inexpensive and noninvasive method compared to CT or DEXA. In the future, it can be a helpful adjunct for increasing the accuracy of diagnosing NAFLD and NASH.

\section{Conflict of interest}

No potential conflict of interest relevant to this article was reported.

\section{Acknowledgments}

We would like to thank Jung Hwa Hong for statistical support.

\section{References}

1. Obesity: preventing and managing the global epidemic. Report of a WHO consultation. World Health Organ Tech Rep Ser 2000;894:i-xii, 1-253.

2. Kim Y. The Korea National Health and Nutrition Examination Survey (KNHANES): current status and challenges. Version 2. Epidemiol Health 2014;36:e2014002.

3. Hedley AA, Ogden CL, Johnson CL, Carroll MD, Curtin LR, Flegal KM. Prevalence of overweight and obesity among US children, adolescents, and adults, 1999-2002. JAMA 2004;291:2847-50.

4. Park YS, Lee DH, Choi JM, Kang YJ, Kim CH. Trend of obesity in school age children in seoul over the past 23 years. Korean J Pediatr 2004;47:247-57.

5. Lakka HM, Lakka TA, Tuomilehto J, Salonen JT. Abdominal obesity is associated with increased risk of acute coronary events in men. Eur Heart J 2002;23:706-13.

6. Clinical guidelines on the identification, evaluation, and treatment of overweight and obesity in adults. WMJ 1998;97:20-1,24-5, 27-37.

7. Hojgaard B, Olsen KR, Sogaard J, Gyrd-Hansen D, Sorensen TI. Obesity related health care costs assessed from BMI or waist circumference: secondary publication. Ugeskr Laeger 2009;171:3068-71.

8. Rössner S, Bo WJ, Hiltbrandt E, Hinson W, Karstaedt N, Santago P, et al. Adipose tissue determinations in cadavers-a comparison between cross-sectional planimetry and computed tomography. Int J Obes 1990;14:893-902.

9. Micklesfield LK, Goedecke JH, Punyanitya M, Wilson KE, Kelly TL. Dual-energy X-ray performs as well as clinical computed tomography for the measurement of visceral fat. Obesity (Silver Spring) 2012;20:1109-14.

10. Armellini F, Zamboni M, Robbi R, Todesco T, Rigo L, Bergamo-Andreis IA, et al. Total and intra-abdominal fat measurements by ultrasound and computerized tomography. Int J Obes Relat Metab Disord 1993;17:20914.

11. Meriño-Ibarra E, Artieda M, Cenarro A, Goicoechea J,
Calvo L, Guallar A, et al. Ultrasonography for the evaluation of visceral fat and the metabolic syndrome. Metabolism 2005;54:1230-5.

12. Cucchi E, Piatti PM, Orena C, Pontiroli AE, Martino E, Paesano PL, et al. Is echography an adequate method for assessing the thickness of intra-abdominal fat? A comparison with computed tomography. Radiol Med 1997;94:329-34.

13. Radikova Z. Assessment of insulin sensitivity/resistance in epidemiological studies. Endocr Regul 2003;37:189-94.

14. Moon JS, Lee SY, Nam CM, Choi JM, Choe BK, Seo JW, et al. 2007 Korean National Growth Charts: review of developmental process and an outlook. Korean J Pediatr 2008;51:1-25.

15. Barlow SE, Dietz WH. Obesity evaluation and treatment: Expert Committee recommendations. The Maternal and Child Health Bureau, Health Resources and Services Administration and the Department of Health and Human Services. Pediatrics 1998;102:E29.

16. Tanda R, Salsberry P. The impact of the 2007 expert committee recommendations on childhood obesity preventive care in primary care settings in the United States. J Pediatr Health Care 2014;28:241-50.

17. Prentice AM, Jebb SA. Beyond body mass index. Obes Rev 2001;2:141-7.

18. Ribeiro-Filho FF, Faria AN, Azjen S, Zanella MT, Ferreira SR. Methods of estimation of visceral fat: advantages of ultrasonography. Obes Res 2003;11:1488-94.

19. Daniels SR. Obesity in the pediatric patient: cardiovascular complications. Prog Pediatr Cardiol 2001;12:161-7.

20. Morrison JA, Sprecher DL, Barton BA, Waclawiw MA, Daniels SR. Overweight, fat patterning, and cardiovascular disease risk factors in black and white girls: The National Heart, Lung, and Blood Institute Growth and Health Study. J Pediatr 1999;135:458-64.

21. Miller J, Rosenbloom A, Silverstein J. Childhood obesity. J Clin Endocrinol Metab 2004:89:4211-8.

22. Daniels SR, Morrison JA, Sprecher DL, Khoury P, Kimball TR. Association of body fat distribution and cardiovascular risk factors in children and adolescents. Circulation 1999;99:541-5

23. Michelson PH, Williams LW, Benjamin DK, Barnato AE. Obesity, inflammation, and asthma severity in childhood: data from the National Health and Nutrition Examination Survey 2001-2004. Ann Allergy Asthma Immunol 2009; 103:381-5.

24. Kim HJ, Cho MH, Park JS, Nam JS, Kang ES, Ahn CW, et al. Visceral fat thickness predicts fatty liver in Koreans with type 2 diabetes mellitus. J Korean Med Sci 2008;23:256-61.

25. Hamagawa K, Matsumura Y, Kubo T, Hayato K, Okawa $\mathrm{M}$, Tanioka $\mathrm{K}$, et al. Abdominal visceral fat thickness measured by ultrasonography predicts the presence and severity of coronary artery disease. Ultrasound Med Biol 2010;36:1769-75.

26. Steinberger J. Diagnosis of the metabolic syndrome in 
children. Curr Opin Lipidol 2003;14:555-9.

27. Kahn SE, Hull RL, Utzschneider KM. Mechanisms linking obesity to insulin resistance and type 2 diabetes. Nature 2006;444:840-6.

28. Motoshima H, Wu X, Sinha MK, Hardy VE, Rosato EL, Barbot DJ, et al. Differential regulation of adiponectin secretion from cultured human omental and subcutaneous adipocytes: effects of insulin and rosiglitazone. J Clin Endocrinol Metab 2002;87:5662-7.

29. Cnop M, Landchild MJ, Vidal J, Havel PJ, Knowles NG, Carr $\mathrm{DR}$, et al. The concurrent accumulation of intra-abdominal and subcutaneous fat explains the association between insulin resistance and plasma leptin concentrations
: distinct metabolic effects of two fat compartments. Diabetes 2002;51:1005-15.

30. Schwimmer JB, Deutsch R, Kahen T, Lavine JE, Stanley C, Behling C. Prevalence of fatty liver in children and adolescents. Pediatrics 2006;118:1388-93.

31. Feldstein AE, Charatcharoenwitthaya P, Treeprasertsuk S, Benson JT, Enders FB, Angulo P. The natural history of nonalcoholic fatty liver disease in children: a follow-up study for up to 20 years. Gut 2009;58:1538-44.

32. Saadeh S, Younossi ZM, Remer EM, Gramlich T, Ong JP, Hurley M, et al. The utility of radiological imaging in nonalcoholic fatty liver disease. Gastroenterology 2002;123: 745-50. 